Results: 712 (out of 33,793 ) transcripts were overexpressed in kidneys from diseased mice (age $>6$ months). These transcripts were significantly enriched in the following pathways: response to type I interferons, antigen processing and presentation, activation and regulation of $B$ cells, complement activation, and regulation of cytotoxicity mediated by $T$ cells

Proportions of renal CD8 T cells with an effector phenotype were significantly increased in the kidneys from 6 months and older compared to younger mice, and compared to paired spleens. Proportions of renal effector CD8 T cells correlated significantly with transcripts involved in interferon signature, adaptive immune responses, cytotoxic T cells, chemotaxis, and correlated negatively with pathways associated with renal tubular cell functions.

Finally, we found a correlation between biological parameters of kidney function (plasma urea, albuminuria) and transcripts involved in pro-fibrotic pathways, chemokines and adaptive immune responses.

Conclusion: Our results confirm the link between the presence of activated immune effectors in the kidney and renal outcomes in a mouse model of SLE, similar to our previous observations in human $\mathrm{LN}$, and warrant further functional studies on the role of kidney-infiltrating $T$ and B cells in this model.

Acknowledgement: This research was funded in part by grants from Fonds de la Recherche Scientifique - FNRS, Fondation Roi Baudouin and Fondation SaintLuc.

Disclosure of Interests: None declared

DOI: 10.1136/annrheumdis-2019-eular.5417

\section{AB0187 CAN ARTIFICIAL INTELLIGENCE REPLACE MANUAL SEARCH FOR SYSTEMATIC LITERATURE REVIEW ON CUTANEOUS MANIFESTATIONS IN PRIMARY SJÖGREN'S SYNDROME?}

Laure Orgeolet $^{1,2}$, Nathan Foulquier ${ }^{1}$, Laurent Misery ${ }^{1}$, Pascal Redou ${ }^{1}$, Jacques Olivier Pers ${ }^{1}$, Valerie Devauchelle-Pensec ${ }^{1}$, Alain Saraux ${ }^{1} .{ }^{1} \mathrm{CHU}$ Brest, Brest, France, ${ }^{2} \mathrm{CHU}$ Brest, Dermatology, Brest, France

Background: Manual systematic literature reviews are becoming increasingly challenging due to the sharp rise in publications. The task is particularly daunting when the study topic is complex.

Objectives: The primary objective of this literature review was to compare manual and in-house computer software retrieval of publications on the cutaneous manifestations of primary Sjogren's Syndrome (pSS). The secondary objective was to evaluate the prevalence of cutaneous manifestations in pSS.

Methods: We compared manual searching and searching with the in-house computer software BIBOT (1) designed for article retrieval and analysis. Both methods were used for a systematic literature review on a complex topic i.e., the cutaneous manifestations of pSS. Articles published in French or English between 1 January 1990 and 30 May 2018 were sought.

Results: The manual search retrieved 855 articles and BIBOT 1042 articles. In all, 202 articles were then selecting by applying exclusion criteria. Among them, 155 were retrieved by both methods, 33 by manual search only, and 14 by BIBOT only. Further selection was performed by reading the 202 articles, of which 54 were deemed relevant, including 23 providing data on the prevalence of one or more cutaneous signs in a cohort of patients with pSS. Cohort sizes and the nature and prevalence of cutaneous manifestations varied across publications. In all, 52 cutaneous manifestations were reported, of which the most common were cutaneous vasculitis (561 patients), xerosis (651 patients), and annular erythema (215 patients).

Conclusion: Agreement was good between the two methods. BIBOT was faster and automatically classified the articles in a chart. Combining the two methods retrieved the largest number of publications. The prevalence of cutaneous manifestations in patients with pSS varied considerably across studies. The advanced machine learning techniques used in artificial intelligence hold promise for literature reviews.

\section{REFERENCES}

[1] Foulquier N, Redou P, Le Gal C, Rouvière B, Pers J-O, Saraux A. Pathogenesis-based treatments in primary Sjogren's syndrome using artificial intelligence and advanced machine learning techniques: a systematic literature review. Hum Vaccin Immunother 2018;14:2553-2558.

Disclosure of Interests: Laure Orgeolet: None declared, Nathan Foulquier: None declared, Laurent Misery: None declared, Pascal Redou: None declared, Jacques-Olivier Pers: None declared, Valerie Devauchelle-Pensec Grant/ research support from: Roche-Chugai, Speakers bureau: MSD, BMS, UCB, Roche, Alain Saraux Consultant for: Roche SAS, Speakers bureau: Chugai Pharma France

DOI: 10.1136/annrheumdis-2019-eular.3981

\section{AB0188 MOLECULAR NETWORKS IN MONOCYTES FROM SYSTEMIC LUPUS ERYTHEMATOSUS PATIENTS RELATED TO THEIR PHYSIOPATHOLOGY. MODULATORY EFFECTS OF ANTI-DSDNA ANTIBODIES AND MOLECULAR MECHANISMS UNDERLYING IN VIVOSTATIN TREATMENT}

Alejandra M. Patiño-Trives ${ }^{1}$, Pérez Sánchez Laura ${ }^{1}$, María Galindo-Izquierdo² ${ }^{2}$ María Luque-Tévar ${ }^{1}$, Alejandro lbañez-Costa ${ }^{1}$, Iván Arias de la Rosa ${ }^{1}$, Maria del Carmen Abalos-Aguilera ${ }^{1}$, Pedro Seguí Azpilcueta ${ }^{1}$, Nuria Barbarroja Puerto', Eduardo Collantes Estevez ${ }^{1}$, Maria A. Aguirre ${ }^{1}$, Carlos Perez-Sanchez ${ }^{1}$, Chary Lopez-Pedrera ${ }^{1}{ }^{1}$ IMIBIC/Reina Sofia Hospital/University of Cordoba, Cordoba, Spain, ${ }^{2}$ Hospital 12 de Octubre, Madrid, Spain

Objectives: 1. To characterize the mRNAs and microRNAs transcriptomes of monocytes from systemic lupus erythematosus (SLE) patients and their association with the pathophysiology of the disease. 2 . To evaluate the role of anti-dsDNA antibodies in the regulation of these processes. 3 . To investigate the molecular mechanisms involved in the efficacy of Fluvastatin in preventing the atherothrombotic risk.

Methods: Monocytes from peripheral blood of 81 SLE patients and 40 healthy donors (HD) were purified by negative immunomagnetic selection. Then, gene expression microarray (Agilent G4112F platform) and nCounter microRNA expression arrays (Nanostring) were performed. Functional categorization of altered genes and miRNAs was made using IPA software, and interaction networks were identified. Genes and miRNAs integrating the networks were validated in the whole cohorts by RT-PCR. Predicted miRNA-mRNA interactions were tested by microRNA over-expression or inhibition experiments. Serum and cellular inflammatory and oxidative profiles were evaluated by multiplex assay, PCR and specific commercial kits, respectively; phosphorylation status of intracellular proteins was analyzed by PathScan array. To evaluate the clinical significance of the parameters analyzed, correlation and association studies were performed. Mechanistic studies were developed to typify the specific effects of the anti-dsDNA antibodies on monocytes. Besides, the beneficial effects of ex vivo Fluvastatin treatment on the monocyte molecular profiles were assessed.

Results: Microarray identified 553 altered genes in SLE monocytes. Relevant biofunctions and disorders on which these genes were involved included inflammatory, immunological, cardiovascular, neurological, renal and reproductive disease. Analysis of miRNA profiles showed altered expression of 35 miRNAs in SLE monocytes. Sixty-one genes were inversely correlated and predicted as CVD-related target genes of 26 differentially expressed miRNAs. Transfection studies confirmed the relationship between specific miRNAs and their identified target genes.

Association of these genes and miRNAs with the anti-dsDNA positivity, early atherosclerosis and nephropathy, along with correlations with disease activity (SLE DAI), activation of some intracellular signaling proteins, and levels of serum inflammatory and oxidative markers were demonstrated. In vitro studies demonstrated the specific modulation of several genes/miRNAs by anti-dsDNA, along with the increase of prothrombotic and proinflammatory mediators, the induction of apoptosis and the phosphorylation of intracellular proteins participating in renal and CVD-related signaling pathways. Besides, treatment of HD-monocytes with SLE patients' serum after Fluvastatin supplementation prevented the proinflammatory altered gene/miRNA profiles induced by serum from those patients before treatment.

Conclusion: 1. Gene and microRNA expression profiles allowed the identification of relevant genes and pathways altered in monocytes of SLE patients, associated with the pathogenesis of the disease, and modulated by anti-dsDNA. 2. Specific microRNA-miRNA regulatory networks control the biological processes and factors related to the CV pathology in SLE, which are prevented by Fluvastatin treatment.

Acknowledgement: Funded by ISCIII, PI15/01333, PI18/00837 and RIER RD16/0012/0015 co-funded with FEDER.

Disclosure of Interests: None declared

DOI: 10.1136/annrheumdis-2019-eular.7142

\section{AB0189 DECREASED URINE SEMAPHORIN 3A SECRETION PREDICTS THE EXTENT OF RENAL DAMAGE IN SYSTEMIC LUPUS ERYTHEMATOSUS PATIENTS}

Doron Rimar ${ }^{1}$, Merav Lidar ${ }^{2}$, Nasrin Eiza ${ }^{3}$, Elias Toubi ${ }^{3}$, Amal Silawy $^{1}$, Gleb Slobodin ${ }^{1}$, Itzhak Rosner ${ }^{1}$, Michael Rosenbaum ${ }^{1}$, Abid Awisat ${ }^{1}$, Nina Boulman ${ }^{1}$, Lisa Kaly ${ }^{1}$, Shira Ginsberg ${ }^{1}$, Nizar Jiries ${ }^{1}$, Zahava Vadasz ${ }^{3} .{ }^{1}$ BnaiZion Medical Center, Rheumatology, Haifa, Israel; ${ }^{2}$ Sheba Medical Center, Rheumatology, Ramat Gan, Israel; ${ }^{3}$ Bnai Zion Medical Center, Division of Allergy and Clinical Immunology, Haifa, Israel

Background: Semaphorins are a family of proteins, involved in axon-guidance, malignancy spread and angiogenesis. Semaphorin $3 \mathrm{~A}$ (sema3A) is recognized also as "immune semaphorin", it is expressed on regulatory T cells and has been 
shown to enhance their inhibitory effect of CD4+ T cell pro-inflammatory function and replication. We have reported a decreased serum levels of sema3A in systemic lupus erythematosus (SLE) compared to healthy controls and in correlation with SLE disease activity [1]. Sema3A was found to be over expressed in podocytes and epithelial cells in in animal models with diabetic nephropathy. Increased urinary sema3A was also detected in diabetic patients with proteinuria and in contrast-induced acute renal injury [2-3].

Objectives: To assess urine sema3A secretion in SLE patients with and without renal involvement compared to rheumatoid arthritis patients as disease control and healthy controls.

Methods: $50 \mathrm{ml}$ of fresh urine samples were collected, centrifuged and the supernatant was then concentrated up to 50 times the initial concentration and subjected to specific human Sema3A ELISA kit (MBS732622, San Diego, CA, USA). Results: Thirty-eight lupus patients fulfilling the 2012 SLICC criteria were recruited, $33(87 \%)$ of whom were women, at a mean age of $35 \pm 12$ years. Eight patients had active nephritis $(21 \%)$ and additional 5 had a history of nephritis but were in remission. APLA was diagnosed in $13(34 \%)$ of patients. Disease activity was evaluated by the Systemic lupus erythematosus disease activity index 2000 (SLEDAI $2 \mathrm{~K}$ ) and was $8.2 \pm 7.7$.

Sema3A was lower in lupus patients compared to rheumatoid arthritis and healthy controls, $4.9 \pm 3.9 \mathrm{ng} / \mathrm{mL}, 8.5 \pm 2.7 \mathrm{ng} / \mathrm{mL}, 9.855 \pm 1.7 \mathrm{ng} / \mathrm{mL}, \mathrm{p}=0.0006$. Lupus nephritis patients demonstrated lower urine sema3A concentration compared to lupus patients without renal involvement $4 \pm 3.4 \mathrm{ng} / \mathrm{ml}, 6.5 \pm 3.8 \mathrm{ng} / \mathrm{ml}, \mathrm{p}=0.03$. Sema $3 A$ reversely correlated with proteinuria $r=-0.43 p=0.006$ and SLEDIA2K $-0.3, p=0.04$, but not with creatinine concentration, disease duration and complement concentration. There was no difference in urinary sema3A between SLE patients with or without APLA syndrome.

Conclusion: Urinary excretion of Sema $3 A$ was found to be decreased in the SLE patients with renal disease, reversely correlating with disease activity and proteinuria. These findings are in line with previous reports of decreased serum level of Sema3A in SLE, that may result in reduced efficacy of regulatory T cells, driving autoimmunity and kidney damage. The discrepancy between low sema3A urinary excretion in SLE nephropathy and increased urinary secretion in other "non- auto immune" conditions with renal damage, suggests that sema3A in the kidneys is protective in autoimmune diseases and detrimental in "non-auto immune" conditions. This differential effect of sema3A may has to do with different populations of effector cells and different expression of sema3A receptors (nuropilin1). Further studies should evaluate semphorin $3 \mathrm{~A}$ role in lupus nephritis and its potential as a treatment option.

\section{REFERENCES}

[1] Vadasz Z, Haj T, Halasz K, Rosner I, et al. Semaphorin 3A is a marker for disease activity and a potential immunoregulator in systemic lupus erythematosus. Arthritis Res Ther;14(3):R146.

[2] Mohamed R, Ranganathan P, Jayakumar C, et al. Urinary semaphorin 3A correlates with diabetic proteinuria and mediates diabetic nephropathy and associated inflammation in mice. J Mol Med (Berl) 2014;92:1245-1256.

[3] Ning L, Li Z, Wei D, et al. Urinary semaphorin 3A as an early biomarker to predict contrast-induced acute kidney injury in patients undergoing percutaneous coronary intervention. Braz J Med Biol Res. 2018;51(4)

Disclosure of Interests: None declared

DOI: 10.1136/annrheumdis-2019-eular.3456

\section{AB0190 CROSSTALK BETWEEN SALIVARY GLAND EPITHELIAL CELLS AND B LYMPHOCYTES IN PRIMARY SJÖGREN'S SYNDROME}

Elodie Rivière $^{1,2}{ }^{\text {, Juliette Pascaud }}{ }^{1}$, Nicolas Tchitchek ${ }^{1}$, Saida Boudaoud ${ }^{1}$, Audrey Paoletti ${ }^{1}$, Bineta Ly ${ }^{1}$,Alice Thai ${ }^{3}$, Norm Allaire ${ }^{3}$, Bernd Jagla ${ }^{4}$, Michael Mingueneau ${ }^{3}$, Gaetane Nocturne ${ }^{1}$, Xavier Mariette ${ }^{1} .{ }^{1}$ Rheumatology, IMVA, CEA - Université Paris Sud - INSERM UMR 1184, Le Kremlin Bicêtre, France; ${ }^{2}$ Arthritis RandD, Paris, France; ${ }^{3}$ Immunology Research, Biogen, Cambridge, United States of America; ${ }^{4}$ Biomarker Discovery Platform UTechS CB, Hub de Bioinformatique et biostatistique C3IB, Institut Pasteur, Paris, France

Background: Primary Sjögren's syndrome (pSS) is an auto-immune disorder characterized by lymphocytic infiltrates and destruction of the salivary glands. Mechanisms leading to $B$ lymphocytes chronic activation remain partially understood and we assumed that salivary gland epithelial cells (SGECs) might play a key role in B lymphocytes activation and differentiation.

Objectives: We aimed to study the interactions between SGECs from pSS patients or controls and B lymphocytes.

Methods: Patients with pSS according to 2016 EULAR/ACR criteria and controls with sicca symptoms were studied. RNASeq analysis was performed on SGECs and B lymphocytes sorted from salivary gland and blood using a FACS ARIA. Enrichment analysis was performed using Ingenuity Pathway Analysis software. Validation of the results was performed by qPCR (Biomark technology). Primary cultured SGECs from pSS patients $(n=6)$ and controls $(n=6)$ were co-cultured with B lymphocytes sorted from healthy donors blood and stimulated with or without PolyIC, INF $\alpha$ or IFNy for 5 days. Transwell assays were performed. Survival, activation (CD38 positivity) and differentiation of $B$ lymphocytes were assessed at day 5 by flow cytometry

Results: The RNASeq analysis of B lymphocytes sorted from salivary gland showed an up-regulation of CD40 and CD48 which are involved in their activation. The analysis of sorted SGECs highlighted IL-7, interferon (IFN) signaling pathways and genes potentially involved in immune responses, including HLA-DRA, BST2 (bone marrow stromal cell antigen 2) and BAFF-R.

Co-culture experiments showed an increase of $B$ lymphocytes viability when cocultured with SGECs compared to B lymphocytes cultured alone $(p<0.05)$. Co-culture with SGECs from pSS compared to controls lead to an increased survival of $B$ lymphocytes (calculated as the percentage of alive co-cultured B lymphocytes percentage of alive cultured alone B lymphocytes) without stimulation or stimulated with Poly $(I: C)(p<0.01)$ (Figure 1A). Similarly, SGECs from $p S S$ were more likely to induce higher activation of B lymphocytes compared to SGECs from controls, assessed by the percentage of CD38+ B lymphocytes (significant after stimulation with Poly $(\mathrm{I}: \mathrm{C})$ ) (Figure 1B). To determine whether the activation of $\mathrm{B}$ lymphocytes and SGECs required a direct cell contact, we used transwell experiments. Preliminary results suggested that the increase of B lymphocytes viability could depend mostly on soluble factors that are currently being identified and will be communicated at the congress.
A

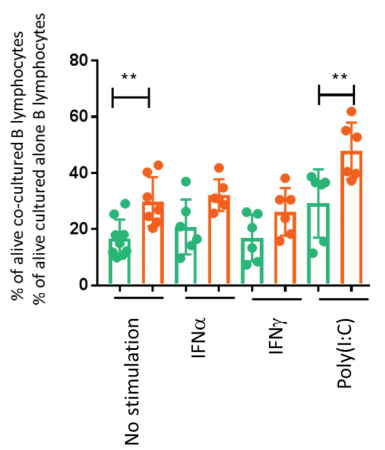

B

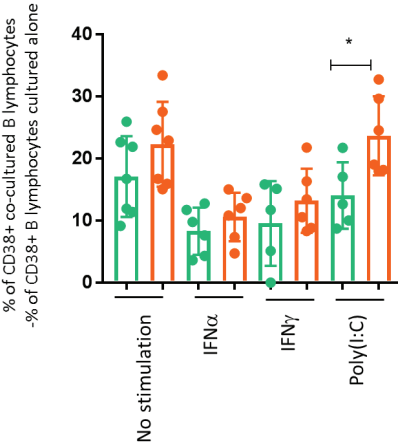

Figure 1. A: Difference between the percentage of alive B lymphocytes co-cultured with SGECs and the percentage of alive B lymphocytes cultured alone at day 5. B: Difference between the percentage of CD38+ $B$ lymphocytes co-cultured with SGECs and the percentage of CD38+ B lymphocytes cultured alone at day 5. In green for SGECs from controls, in orange for SGECs from pSS patients. One asterisk indicates $p$-value $<0.05$, two asterisks indicate $\mathrm{p}$ value $<0.01$.

Conclusion: Epithelial cells from patients with pSS have better ability than controls to stimulate survival and activation of B cells. According to preliminary results, this effect could be mediated at least partially by soluble factors. The pathways responsible for this stimulation are being determined by RNASeq analysis of purified epithelial cells and $B$ lymphocytes and inhibitory experiments that can represent new therapeutic perspectives are ongoing.

Disclosure of Interests: Elodie Rivière: None declared, Juliette Pascaud: None declared, Nicolas Tchitchek: None declared, Saida Boudaoud: None declared, Audrey Paoletti: None declared, Bineta Ly: None declared, Alice Thai Employee of: Alice Thai is employed by Biogen., Norm Allaire Employee of: Norm Allaire is employed by Biogen., Bernd Jagla: None declared, Michael Mingueneau Employee of: Michael Mingueneau is employed by Biogen., Gaetane Nocturne: None declared, Xavier Mariette Grant/research support from: Servier, Consultant for: AstraZeneca, Bristol-Myers Squibb, GlaxoSmithKline, Janssen, Pfizer, UCB Pharma DOI: 10.1136/annrheumdis-2019-eular.4121

\section{AB0191 ENDOTHELIN-1 LEVEL IN PATIENTS WITH ANTIPHOSPHOLIPID SYNDROME, ITS ASSOCIATION WITH ENDOTHELIAL DYSFUNCTION AND ATHEROSCLEROSIS}

Sergii Shevchuk ${ }^{1}$, Inna Kuvikova ${ }^{2}$, Yuliia Seheda ${ }^{3}$, Olena Galiutina ${ }^{2} .{ }^{1}$ National Pirogov Memorial Medical University, Vinnitsya, Ukraine; ${ }^{2}$ Scientific and Research Institute of Invalid Rehabilitation on the base of National Pirogov Memorial Medical University, rheumatology, Vinnitsya, Ukraine; ${ }^{2}$ Scientific and Research Institute of Invalid Rehabilitation on the base of National Pirogov Memorial Medical University, rheumatology, Vinnitsya, Ukraine

Background: It is known that endothelin-1 is one of the leading factors in the development of coronary artery disease, acute myocardial infarction, atherosclerosis of cerebral and peripheral vessels, pulmonary hypertension, ischemic lesion 